\title{
CONTROL OF PHOTO-ELECTRON-TRANSFER INDUCED RADICAL PRODUCTION BY MICELLAR CAGES, HEAVY-ATOM SUBSTITUENTS AND MAGNETIC FIELDS
}

\author{
T. UlRich and U. E. STEINER* \\ Fakultät für Chemie, Universität Konstanz, Postfach 5560, D-7750 Konstanz, \\ Federal Republic of Germany \\ and \\ W. SCHLENKER \\ Institut für Physikalische Chemie der Universität Stuttgart, Pfaffenwaldring 55, \\ D-7000 Stuttgart 80, Federal Republic of Germany
}

\begin{abstract}
Intramicellar radical pair formation and recombination kinetics in the electron transfer quenching of the thionine triplet by aniline and various monohalogenated anilines have been studied by microsecond and nanosecond laser flash spectroscopy in reversed micellar solution of CDBA in benzene. Clear kinetic evidence of the micellar cage effect is provided by a comparative spectro-kinetical study in homogeneous aqueous and reversed micellar solution. In zero magnetic field the radical pairs. which originate with a triplet spin alignment recombine in the waterpools of the micelles with a rate constant of about $3 \times 10^{6} \mathrm{~s}^{-1}$ which is not sensitive to the hyperfine or spin-orbit coupling parameters of the anilinetype radical. Long lived radicals are formed by radical escape from the micelles occurring with a rate constant in the order of $2 \times 10^{6} \mathrm{~s}^{-1}$ and being insensitive to an external magnetic field. Intramicellar radical pair recombination is slowed down by an external magnetic field. A maximum effect (measured at $1 \mathrm{~T}$ ) of a factor of 3 is observed for non-halogenated anilines. Halogen substitution attenuates this magnetic-field effect depending on the strength of spin-orbit coupling exhibited by the halogen substituent. The magneticfield effect is interpreted in terms of the radical pair mechanism with special emphasis on the role of spin relaxation. Suppression of the magnetic-field effect by halogen substituents is attributed to the spin-rotational relaxation mechanism which is independent of a magnetic field. A heavy-atom substituent effect is also borne out in the primary yield of radical pairs which is decreased in the same way as in homogeneous solution. This is attributed to the role of a triplet exciplex formed as a precursor of the radical pair, where heavy-atom substituents cause very efficient radiationless decay to the ground state. A magnetic-field effect typical for the triplet mechanism in the exciplex has been detectable with 4-iodoaniline as quencher.
\end{abstract}

Among the various mechanistic pathways of photochemical radical production photo-induced electron transfer is a most important one, especially if the generation of radical ions is concerned. The radical pairs produced in this way are generally higher in energy than the corresponding ground state unreacted donor-acceptor pairs and therefore may be stabilized by fast reverse electron transfer before the radicals can separate. The efficiency of reverse electron transfer vs radical separation determines the yield of free radicals and is hence of great importance, if one is interested to utilize the radicals for further chemical transformations, e.g. for the purpose of chemical storage of solar energy or information or for chemical synthesis.

One of the basic principles, which has been implicitly confirmed in many investigations, is the conservation of electron spin in the electron transfer step. In fact, spin conservation is quite general in fast chemical reactions and in connection with the production or recombination of radical pairs it is the basic requirement of the mechanisms explaining chemical polarization of nuclear and electronic spins (CIDNP, CIDEP) ${ }^{1,2}$ and of various magnetic-field effects in chemical reactions (for reviews cf. Refs 36). Thus, when excited singlets react with closed-shell electron donors or acceptors, radical pairs in an overall singlet state are formed, i.e. the radicals originate with antiparallel spin alignment. Correspondingly, when starting from an electronically excited triplet state the radicals will originate with parallel spin alignment. As a consequence of the rule of spin conservation in electron transfer, direct recombination of triplet radical pairs to form diamagnetic ground state products is spin forbidden. Due to this fact the free radical yields observed in excited triplet state reactions are generally much higher than for the corresponding singlet state reactions. ${ }^{7-10}$ of course, the principle of electron spin conservation is an idealization and the rule can be relaxed due to several types of perturbation mechanisms, which are also responsible for the magnetic polarization effects mentioned above.

During the last years we have been especially interested in the mechanisms and rules governing "spinforbidden" electron back transfer following electron transfer reactions with excited triplet states. A number of general conclusions has been obtained from our studies ${ }^{8,11-13}$ of the reaction of the thionine triplet $\left({ }^{3} \mathrm{TH}^{+}\right)$with aniline and its monohalogenated derivatives, where the dye triplet acts as an electron acceptor.

The general mechanistic aspects may be described in terms of Scheme 1, where A stands for electron acceptor and D for electron donor and the charges correspond to the systems we have studied. According to Scheme 1 a radical pair like triplet exciplex has to be considered as the primary product of the electron<smiles>Nc1ccc2nc3ccc(=[NH2+])cc-3sc2c1</smiles> 


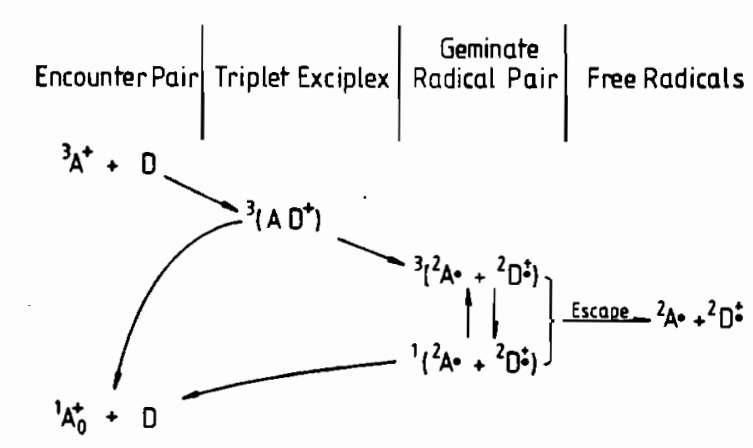

Scheme 1. Pathways of "spin forbidden" reverse electron transfer.

transfer. It may dissociate to yield a radical pair with individually solvated radicals which are free to diffuse apart or to undergo several random re-encounters. This radical pair is generally termed geminate radical pair. Two routes of fast electron back transfer may be discriminated. $\dagger$

(1) Directly from the triplet exciplex, which might be conceived as a kind of intramolecular intersystem crossing process. It does not necessarily occur via the intermediacy of the singlet exciplex.

(2) From the geminate pair.

We have found that there is a very systematic, position-dependent heavy atom effect on the free radical yield when monosubstituting aniline by various halogens. " The analysis of these results has led us to the conclusion that the efficiency of electron back transfer from the geminate radical pair is negligible in solvents of normal viscosity for radical pairs without Coulomb attraction. On the other hand, if spin-orbit coupling is strong enough in the triplet exciplex, which can be achieved by means of heavy atom substitution, its intersystem crossing to the ground state, namely "spin-forbidden reverse electron transfer", can effectively compete with its dissociative decay and hence decrease the radical yield.

The intermediacy of the triplet exciplex in the course of radical formation has been corroborated by a magnetic-field effect. ${ }^{13}$ The free radical yield is decreased by a magnetic field, the effects being especially pronounced for systems with strong spin-orbit coupling. The negative sign of this effect and the typical halffield value of about $200 \mathrm{mT}$ exclude the possibility that the geminate radical pair may be responsible for this effect. A consistent explanation is provided, however, by the so-called triplet mechanism (Scheme 2 ). The heavy atom enhancement of spin-orbit coupling causes a sublevel-selective intersystem crossing from triplet exciplex to singlet ground state. Therefore, one of the sublevels $\left(T_{z}\right)$ because of its less efficient intersystem crossing contributes predominantly to the radical yield. The magnetic field, however, destroys the sublevel selectivity of intersystem crossing and thus, indirectly, decreases the radical yield. From a quantitative evaluation of the magnetic field effect the dissociative lifetime of these

†The analogous case of spin inverted backward electron transfer, however with $\mathrm{S} \rightarrow \mathrm{T}$ spin conversion, has been discussed by Weller ${ }^{14}$ using a kinetic scheme similar to our Scheme 1.

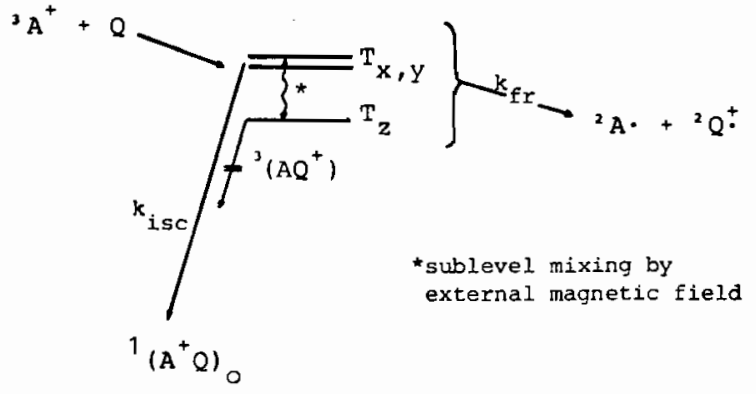

Scheme 2. Triplet mechanism.

- triplet exciplexes could be estimated to be in the order of 200 ps.

Without the heavy atom effect the radical yield in the thionine triplet/aniline system is close to 1 in homogeneous solution, ${ }^{11}$ showing that neither in the triplet exciplex nor in the geminate radical pair electron back transfer can efficiently compete with radical separation. If, however, the cage lifetime of the geminate radical pair is increased, which can be achieved by using micellar solubilization, the yield of free radicals may be considerably decreased even without heavy atom substituents. ${ }^{15}$ Large micellar cage effects on the recombination yield of radical pairs were first reported by Turro and co-workers ${ }^{16}$ who also found magnetic field effects modifying the micellar cage effect. ${ }^{17}$ Meanwhile it has been possible to directly time resolve intramicellar recombination for some radical pairs derived from carbonyl triplet reactions, demonstrating that this recombination is slowed down by a magnetic field. ${ }^{18-21}$ These results have been generally discussed in terms of the radical pair mechanism which has been introduced to explain CIDNP ${ }^{22,23}$ and has been further elucidated by time-resolved laserflash-spectroscopy experiments ${ }^{24,25}$ and by sophisticated theoretical treatments. $3,26,27$

The characteristic features of our investigations on radicals in micellar systems are that:

(a) they deal with reversed micelles, which have the detergent molecules with their polar head groups oriented to the inside where a sizeable waterpool may be enclosed and

(b) electron transfer is used to produce radical pairs.

Recently we reported time-resolved measurements of the recombination kinetics of such electron-transfer derived radical pairs in reversed micelles. ${ }^{28}$ Depending on the size of the micelles, recombination occurs at a rate of $5 \times 10^{6}-2 \times 10^{5} \mathrm{~s}^{-1}$. By applying external magnetic fields this rate can be slowed down to almost one-third of its zero field value. Our previous results have provided strong evidence that in reversed micelles-and probably in normal micelles, too-the radical pair mechanism as normally applied in homogeneous solution (cf. e.g. Refs 23-27) is not sufficient for a quantitative understanding. Due to the long cage lifetime, the role of spin relaxation becomes essential. Apparently this feature, which was first pointed out by Hayashi and Nagakura, ${ }^{29}$ has not yet been generally recognized.

In this paper we report further experimental results pertaining to the interesting question of intramicellar recombination of spin-correlated radical pairs. We first give a kinetic documentation of the micellar cage effect as compared to homogeneous aqueous solution, 
secondly we provide further evidence that in zero field spin relaxation is faster than the observed recombination and hence not rate determining for this process, and thirdly we investigate the effect of heavy atom substituents on intramicellar radical pair production efficiency and recombination kinetics as well as their magnetic field dependence.

\section{RESULTS}

For preparing reversed micellar solutions of thionine we used cetyldimethylbenzylammonium chloride (CDBA) in benzene with a suitable amount of water (cf. Experimental). This reversed micellar system has been introduced by Hauser and co-workers. ${ }^{30}$ With the particular water concentration used in this work, the aggregation number of the micelles is about 150 , corresponding to a concentration of micelles of $2.7 \times 10^{-4} \mathrm{~mol} \mathrm{l}^{-1}$ and about 2000 molecules of water solubilized in one micelle. The dye concentration was not higher than $5 \times 10^{-6} \mathrm{~mol} \mathrm{l}^{-1}$ so that the mean occupation number of micelles by dye molecules was below 0.02 .

The absorption spectra of the thionine ground state $\left(\mathrm{TH}^{+}\right)$, triplet $\left({ }^{3} \mathrm{TH}^{+}\right)$and reduced dye radical $\left({ }^{2} \mathrm{TH}^{*}\right)$, produced when the triplet is quenched by aniline are shown in Fig. 1. They are rather similar to the corresponding spectra obtained in homogeneous solvents as methanol or water., ${ }^{81}$ The long wavelength maximum of the triplet absorption is shifted to the red by about $15 \mathrm{~nm}$ as compared to the solvents mentioned above. The absorption band of the radicals centred at $420 \mathrm{~nm}$ is mainly due to the semithionine $\left({ }^{2} \mathrm{TH}^{\circ}\right)$. Aniline radical cation absorption ${ }^{32}$ in this wavelength region probably does not contribute more than $15 \%$.

Kinetic evidence of the cage effect in reversed micelles

In order to assess the specific effect of micellar solubilization of the dye on the transient kinetics, we performed parallel experiments comparing thionine triplet quenching by aniline in homogeneous aqueous and in reversed micellar solution. The transient signals were recorded at three characteristic wavelengths (indicated by the arrows in Fig. 1) namely 780 (observation of the triplet), 600 (observation of thionine ground state bleaching and recovery) and $420 \mathrm{~nm}$ (simultaneous observation of triplet and dye radical). The results are presented in Fig. 2.

The triplet decay curves at $780 \mathrm{~nm}$ show that the triplet is dynamically quenched in both solvents. The quenching constants evaluated from these measurements are $7 \times 10^{9} 1 \mathrm{~mol}^{-1} \mathrm{~s}^{-1}$ in aqueous and $1.4 \times 10^{9} \mathrm{~mol}^{-1} \mathrm{~s}^{-1}$ in reversed micellar solution, showing that quenching is somewhat slower in the micellar system.

Unlike the observations at $780 \mathrm{~nm}$, the transient kinetics at $600 \mathrm{~nm}$, where the transient bleaching of the solution is observed, is rather different in homogeneous and reversed micellar solution. On the water side we see that an absorbance recovery goes along with the triplet quenching. After triplet decay is complete, about $25 \%$ of the initial absorbance has been recovered. The time constant of recovery for the bleaching, remaining after $2000 \mathrm{~ns}$, is in the order of milliseconds. The fast absorbance recovery, exactly paralleling triplet decay, may be attributed to the formation of semithionine radicals by electron transfer from aniline to the thionine triplet. The increase of absorption during the triplet decay is mainly due to the appearance of semithionine and aniline cation radical absorption and, to a lesser degree, to the thionine ground state repopulation.

In micellar solution the absorbance recovery at $600 \mathrm{~nm}$ follows the triplet decay kinetics only at low quencher concentrations. It approaches, however, constant decay kinetics with a time constant of about $200 \mathrm{ns,}$ which is observed even when the triplet lifetime is already below $10 \mathrm{~ns}$. This means that with high quencher concentrations the time-resolved absorbance recovery cannot be due to the growing-in of the radical absorption, which should occur at the same rate constant as the triplet decay. A reasonable explanation, however, of the limiting rate constant of absorption recovery at high quencher concentrations is to assign it to fast radical recombination, leading to ground state repopulation. The slow part of the absorbance recovery (also in the order of milliseconds) should then correspond to radicals which may have escaped the micellar cage and are only intercepted by homogeneous second-order recombination. It should be noted that with quencher concentrations higher than $5 \times 10^{-3}$ mol $1^{-1}$ fluorescence quenching is not negligible. Thus the amount of initially formed radicals decreases as the quencher concentration is increased.

The observations at $425 \mathrm{~nm}$ corroborate the interpretation given for the $600 \mathrm{~nm}$ kinetics. Since triplet absorption and radical absorption are approximatly equal at this wavlength, there is no effect of the quencher to be seen in aqueous solutions, since

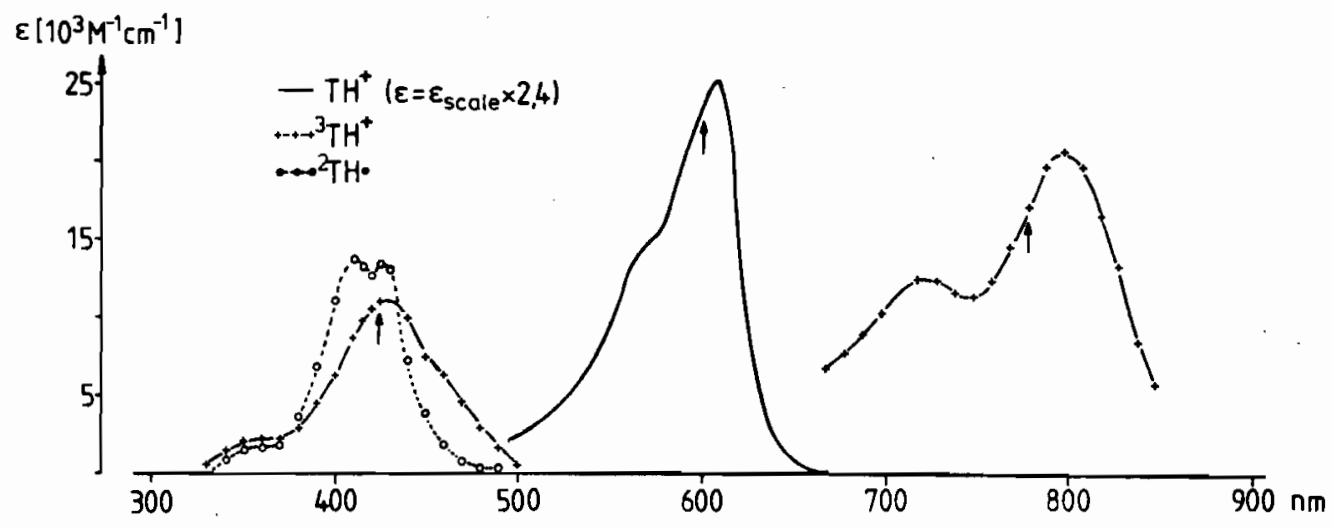

Fig. 1. Absorption spectra of thionine $\left(\mathrm{TH}^{+}\right)$, thionine triplet $\left({ }^{3} \mathrm{TH}^{+}\right)$and semireduced thionine $\left({ }^{2} \mathrm{TH}^{*}\right.$, produced by triplet quenching with aniline) in reversed micellar solution. 

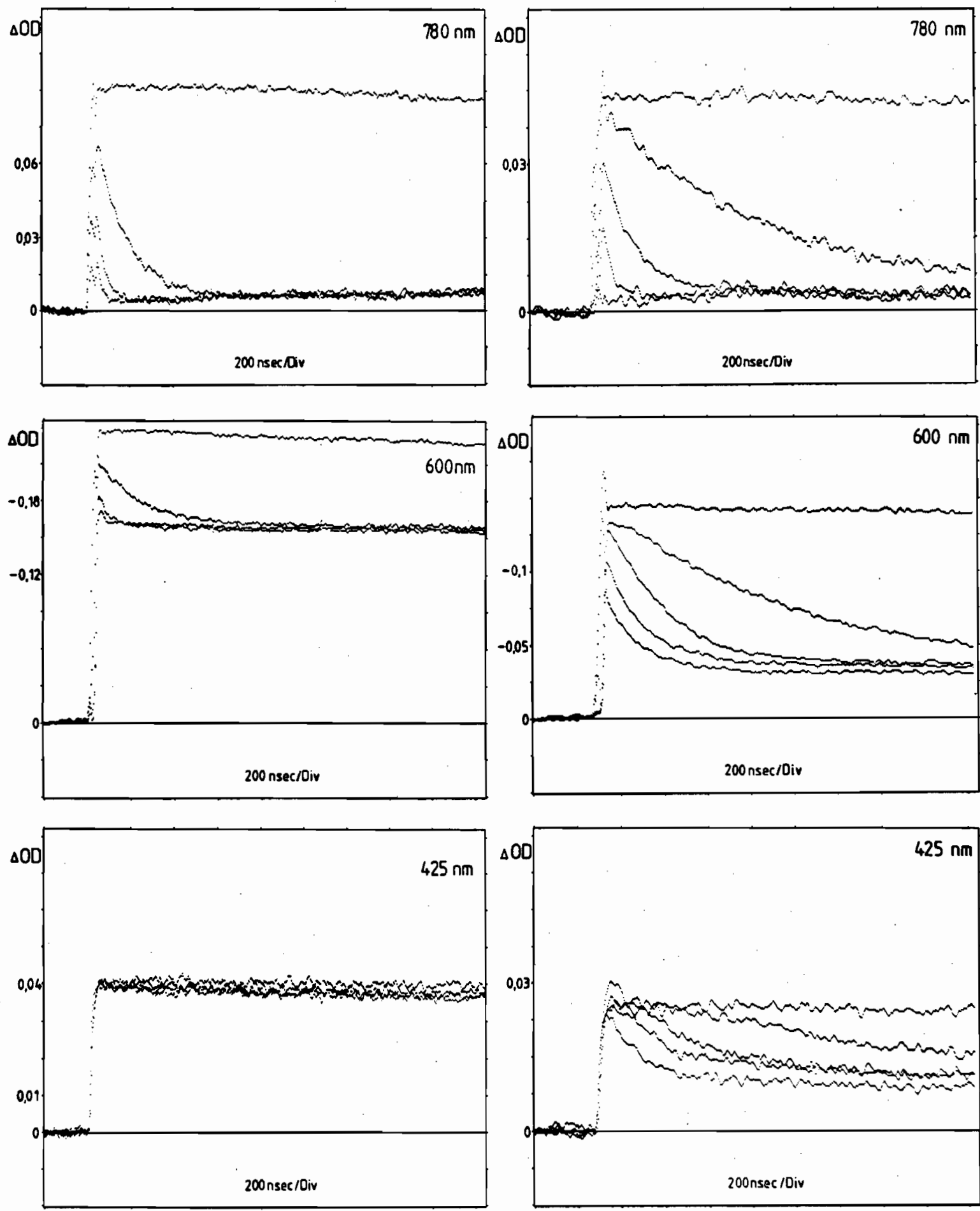

Fig. 2. Transient kinetics of thionine in the presence of various concentrations of aniline in water (lefthand side, $c_{\mathrm{AN}}=0,10^{-3}, 5 \times 10^{-3}$ and $10^{-2} \mathrm{~mol} \mathrm{l}^{-1}$ ) and in reversed micellar solution (right-hand side, $c_{\mathrm{Aa}}=0,10^{-3}, 5 \times 10^{-3}, 2 \times 10^{-2}$ and $10^{-1} \mathrm{~mol} 1^{-1}$ ). The concentration of thionine is $5 \times 10^{-6} \mathrm{~mol} 1^{-1}$ in both cases. In each diagram the order of the signals from top to bottom at $800 \mathrm{~ns}$ delay time corresponds to increasing concentration of the quencher.

transformation of the triplet to the radical is not expressed as an absorbance change at this wavelength. However, at $425 \mathrm{~nm}$ in micellar solution the absorbance decays at an increasing rate with increasing quencher conentration, but finally reaches a limiting value corresponding to the time constant of about $200 \mathrm{~ns}$ already observed a $600 \mathrm{~nm}$. The fluorescence quenching effect on the initial radical yield is also detectable in the amplitudes of the signals.
In summarizing the conclusions from Fig. 2 we can say that, although triplet quenching occurs similarly in homogeneous and reversed micellar solutions, the radical decay kinetics are markedly different. Whereas in homogeneous aqueous solution there is nothing like a fast geminate recombination of radicals to be observed, in reversed micellar solution there is clearly a two-step radical recombination where we attribute the fast one to intramicellar geminate radical recom- 


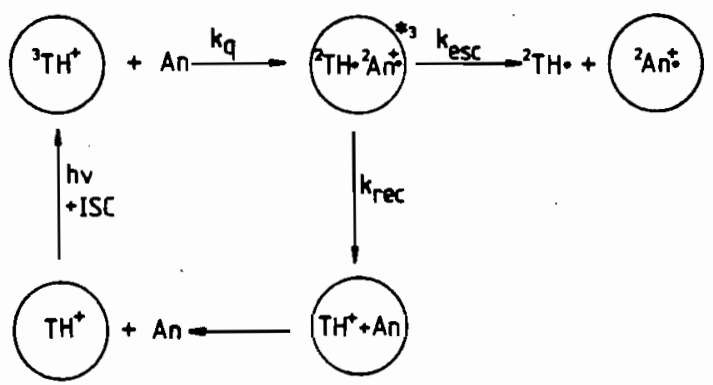

Fig. 3. Reaction scheme for thionine triplet $\left({ }^{3} \mathrm{TH}^{+}\right)$and aniline (An) in reversed micellar solution. The circles indicate the waterpools of the reversed micelles. The symbol *3 denotes that the radical pair is created with parallel spins.

bination and the slow one to the normal homogeneous recombination process of radicals which have escaped the micelles. An appropriate reaction scheme describing these observations in reversed micellar solution is depicted in Fig. 3. Here we have tentatively assumed that it is the neutral semiquinone radical $\mathrm{TH}^{*}$ which escapes from the waterpool into the bulk solvent.

\section{Microsecond time-resolved experiments on the yield of free radicals}

In order to determine the yield of free radicals in relation to the amount of triplets, initially produced, the ground state bleaching kinetics was observed at $600 \mathrm{~nm}$, using quencher concentrations such that the triplet lifetime was reduced to about $5 \mu$ s. Since the unquenched triplet lifetime is about $150 \mu$ s the triplet decay is still almost due to bimolecular quenching. On the other hand, in the signals observed the amplitude (maximum bleaching at the end of the $0.8 \mu$ s laser pulse used for these experiments) corresponds to a situation where most of the triplet is still unquenched. From the ratio of bleaching maximum to the remaining bleaching after complete triplet decay we have evaluated approximate values for the yield of free radicals in the triplet quenching. The results obtained in this way with a series of monohalogenated anilines are collected in Table 1. Also given are the triplet quenching rate constants found with these donors in reversed micelles.

Comparing the results with those in homogeneous methanolic solution we find that quenching by aniline

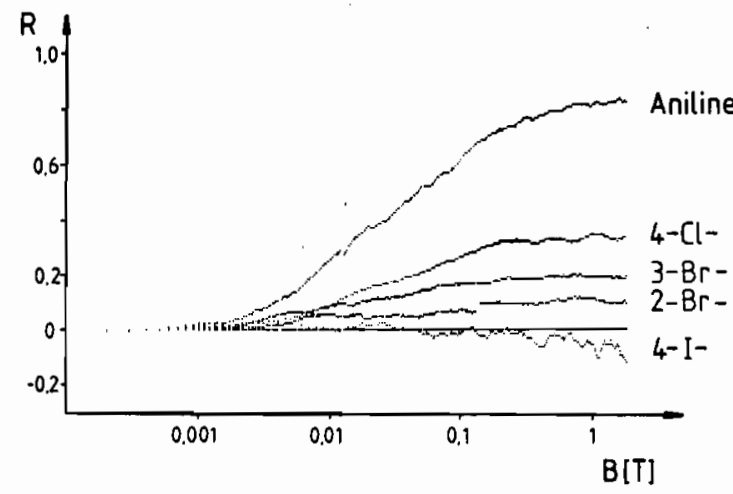

Fig. 4. Relative magnetic field effect $R$ (with respect to zero field) of the free radical yield produced in thionine triplet quenching with various anilines and monitored $20 \mu$ s after the laser pulse. The magnetic field was scanned continuously over 480 pulses. Each data point represents an average of 30 pulses.

and the 3- and 4-substituted halogenanilines is about 2.5 times slower in micellar solution than in methanol. However, 2-substitution leads to an 8-fold reduction in the rate constant of quenching. Steric differences might be more important in the microheterogeneous environment than in homogeneous solution.

The heavy atom effect on the free radical yield which is documented by the data from homogeneous solvent does also show up in the reversed micellar solution experiments. Apart from the effect that the absolute values are significantly smaller in micelles than in homogeneous solutions their relative values are not much affected by the change of the medium. Also, the position dependence is borne out by the bromoaniline data.

As we have previously shown with aniline, the free radical yield in reversed micellar systems can be strongly enhanced by applying an external magnetic field. ${ }^{15}$ In the present work the free radical yield has been also determined as a function of the magnetic field for some of the substituted anilines listed in Table 1. Figure 4 shows the results of these measurements, which have been obtained by sampling the groundstate bleaching at $20 \mu$ s delay time with a special microprocessor-controlled technique (cf. Experimental and Ref. 15). The figure documents, that in the system with aniline as quencher the free radical

Table 1. Thionine triplet quenching by anilines: quenching rate constants $\left(k_{\mathrm{q}}\right)$, free radical yields $\left(\Phi_{\mathrm{fr}}\right)$ and magnetic field effects $(R)$ on $\Phi_{\mathrm{fr}}$

\begin{tabular}{|c|c|c|c|c|c|c|}
\hline & \multicolumn{3}{|c|}{ In methanol } & \multicolumn{3}{|c|}{ In reversed micelles } \\
\hline & $k_{\mathrm{q}}^{a, b}$ & $\Phi_{\mathrm{fr}}^{a}$ & $R^{c}$ & $k_{\mathrm{q}}^{b}$ & $\Phi_{\mathrm{fr}}$ & $R^{c}$ \\
\hline Aniline & 3.4 & 1.00 & \pm 0 & 1.4 & 0.29 & +90 \\
\hline $4-\mathrm{Cl}$ & 2.6 & 0.97 & \pm 0 & 1.4 & 0.27 & +34 \\
\hline $3-\mathrm{Br}$ & 1.0 & 0.90 & \pm 0 & 0.4 & 0.21 & +20 \\
\hline $2-\mathrm{Br}$ & 0.8 & 0.70 & \pm 0 & 0.1 & 0.13 & +10 \\
\hline $4-\mathrm{Br}$ & 2.8 & 0.51 & -2 & 1.3 & 0.10 & \pm 0 \\
\hline $4-I$ & 3.7 & 0.13 & -22 & .1 .5 & 0.06 & -10 \\
\hline
\end{tabular}


Table 2. Magnetic field dependent intramicellar radical pair recombination : rate constants of escape $\left(k_{\text {esc }}\right)$ and recombination $\left(k_{\text {rec }}\right)$

\begin{tabular}{lcccccc}
\hline & $k_{\mathrm{esc}}{ }^{a, b}$ & $k_{\mathrm{rec}}{ }^{a}(B=0 \mathrm{~T})$ & $k_{\mathrm{rec}}{ }^{a}(B=1 \mathrm{~T})$ & $f^{\boldsymbol{c}}$ & $B_{1 / 2}{ }^{d}(\exp )$ & $B_{1 / 2}{ }^{e}(\mathrm{IHF})$ \\
\hline Aniline & 1.7 & 3.0 & 0.94 & 3.2 & 14 & 3.0 \\
N,N-Dimethyl & 1.4 & 2.4 & 0.80 & 3.0 & 17 & 5.2 \\
4-F & 1.9 & 2.4 & 0.96 & 2.5 & 50 & 4.0 \\
$4-\mathrm{Cl}$ & 1.7 & 3.0 & 1.58 & 1.9 & 20 & 3.0 \\
$3-\mathrm{Br}$ & 2.9 & 3.1 & 2.1 & 1.5 & 10 & 3.0 \\
$4-\mathrm{Br}$ & 2.9 & 3.4 & 3.4 & 1.0 & & \\
\hline
\end{tabular}

${ }^{a} 10^{6} \mathrm{~s}^{-1}$

${ }^{b}$ Magnetic field independent.

${ }^{c} f=k_{\text {rec }}(B=0 \mathrm{~T}) / k_{\text {rec }}(B=1 \mathrm{~T})$.

${ }^{d} \mathrm{In} \mathrm{mT}$, interpolated field value giving average of $k_{\mathrm{rec}}(B=0 \mathrm{~T})$ and $k_{\mathrm{rec}}(B=1 \mathrm{~T})$.

${ }^{\mathrm{e}} \mathrm{In} \mathrm{mT}$, calculated according to Eq. (5).

yield is most strongly sensitive to a magnetic field. The size of the magnetic field effect decreases in the series 4chloro-, 3-bromo-, 2-bromoaniline and is completely absent for 4-bromoaniline (not included in Fig. 4). For 4-iodoaniline the sign of the effect has been inverted, now corresponding to a decrease of the radical yield by a magnetic field. There is also a significant change of the half-field value which accompanies the sign inversion. For the systems where the magnetic field effect on the radical yield shows a positive sign, the half-field values are in the order of $20-30 \mathrm{mT}$ whereas for the 4-iodoaniline system it is in the order of $300-400 \mathrm{mT}$.

The $R$ values for the magnetic field effects ( $R$ being defined as the relative change of the radical yield compared with the zero field radical yield) obtained at the highest field strength applied $(1.8 \mathrm{mT})$ are also listed in Table 1 . Whereas in homogeneous solution only the negative type of magnetic field effect is observed, requiring donors which exhibit a strong heavy atom effect on the absolute yield of free radicals, the positive type of magnetic field effect, particularly exemplified by the aniline case, is characteristic of the reversed micellar system. This magnetic field effect is quenched if halogen substituents are introduced and it should be noted that the efficiency in quenching the magnetic field effect parallels the heavy atom effect on the free radical yield at zero field. The inverted magnetic field effect found for the 4-iodoaniline quencher case in reversed micellar solution (cf. Table 1) corresponds to the effect observed in methanol. However, it is somewhat smaller than in homogeneous solution.

Nanosecond laser flash experiments. The intramicellar recombination kinetics and its magnetic field dependence have been measured for a series of aniline derivatives. The observed decay curves at several values of the magnetic field are displayed in Fig. 5. The

\footnotetext{
$\dagger$ In the Tektronix $7912 \mathrm{AD}$ digitizer, $\mathrm{AD}$ conversion is not directly performed from the input signal but from a charge trace produced by sweeping an electron beam over a multidiode target. For optimum resolution of the charge trace the electron beam intensity has to be adjusted depending on horizontal and vertical sweep rate. In the signals displayed in Fig. 5 the electron beam intensity was adjusted to give optimal performance in the time region of radical decay, where the magnetic field effect appears. So it was somewhat too weak to yield proper digitization in the first $50 \mathrm{~ns}$ after the laser pulse where horizontal and vertical sweep rate are high in the case of rapid triplet-ground state conversion.
}

quencher concentrations applied in each case were chosen so as to bring the effective triplet lifetime into the order of the laser pulse width (15-20 ns), using donor concentrations of $0.05 \mathrm{M}$ (aniline, 4-fluoro-, 4bromo-, 4-iodoaniline) or $0.5 \mathrm{M}$ (N,N-dimethylaniline, 3-bromoaniline).

Due to the short triplet lifetime the signal amplitudes at $600 \mathrm{~nm}$, where ground state bleaching is monitored, are an approximate measure of the total amount of dye semiquinone radicals produced from the triplets. Since the laser-pulse energy was approximately constant throughout the series the signal amplitudes in Fig. 5 should qualitatively reflect the yield of geminate radicals produced in the triplet quenching. In going through the series from aniline to 4-bromoaniline we note that, while the geminate radical yield is highest for aniline, N,N-dimethylaniline and 4-fluoroaniline, there is a marked decrease in the series 4-chloroaniline, 3-bromoaniline and 4bromoaniline. We note that, qualitatively, the yield of geminate radical pairs to be observed shortly after the laser pulse follows the yield of free radicals listed in Table 1. This trend has also been verified for 4 iodoaniline, however, in this case the signal is already too small to be measured with sufficient accuracy in the nanosecond apparatus.

Comparing the kinetics of intramicellar radical pair recombination at zero field (lowest signal trace in each diagram) we find that there is practically no difference between aniline, N,N-dimethylaniline and 4-fluoroaniline. When analyzing the decay kinetics in terms of the scheme given in Fig. 3, rate constants of escape and intramicellar recombination can be obtained by a numerical fit according to

$$
\begin{aligned}
& c_{\mathrm{TH} \cdot}(t)=c_{\mathrm{TH} \cdot}(0)\left[k_{\mathrm{esc}} /\left(k_{\mathrm{esc}}+k_{\mathrm{rec}}\right)\right] \\
& \times\left[1+\left(k_{\mathrm{rec}} / k_{\mathrm{esc}}\right) \exp \left\{-\left(k_{\mathrm{esc}}+k_{\mathrm{rec}}\right) t\right\}\right] .
\end{aligned}
$$

The decay of the free radicals is not considered on the time scale of interest. Hence monoexponential decay kinetics are expected. In fact, for aniline, N,Ndimethylaniline and 4-fluoroaniline such a monoexponential decay fits the observed signals very well. The results are given in Table 2. However, when going to the heavier halogen substituents an initial fast decay component becomes more and more apparent.

At present we suppose that this fast component is due to ground state repopulation from the triplet, which may appear somewhat longer than it really is, due to the performance characteristics of the transient digitizer. $\dagger$ This fast kinetics is hidden if the conversion 

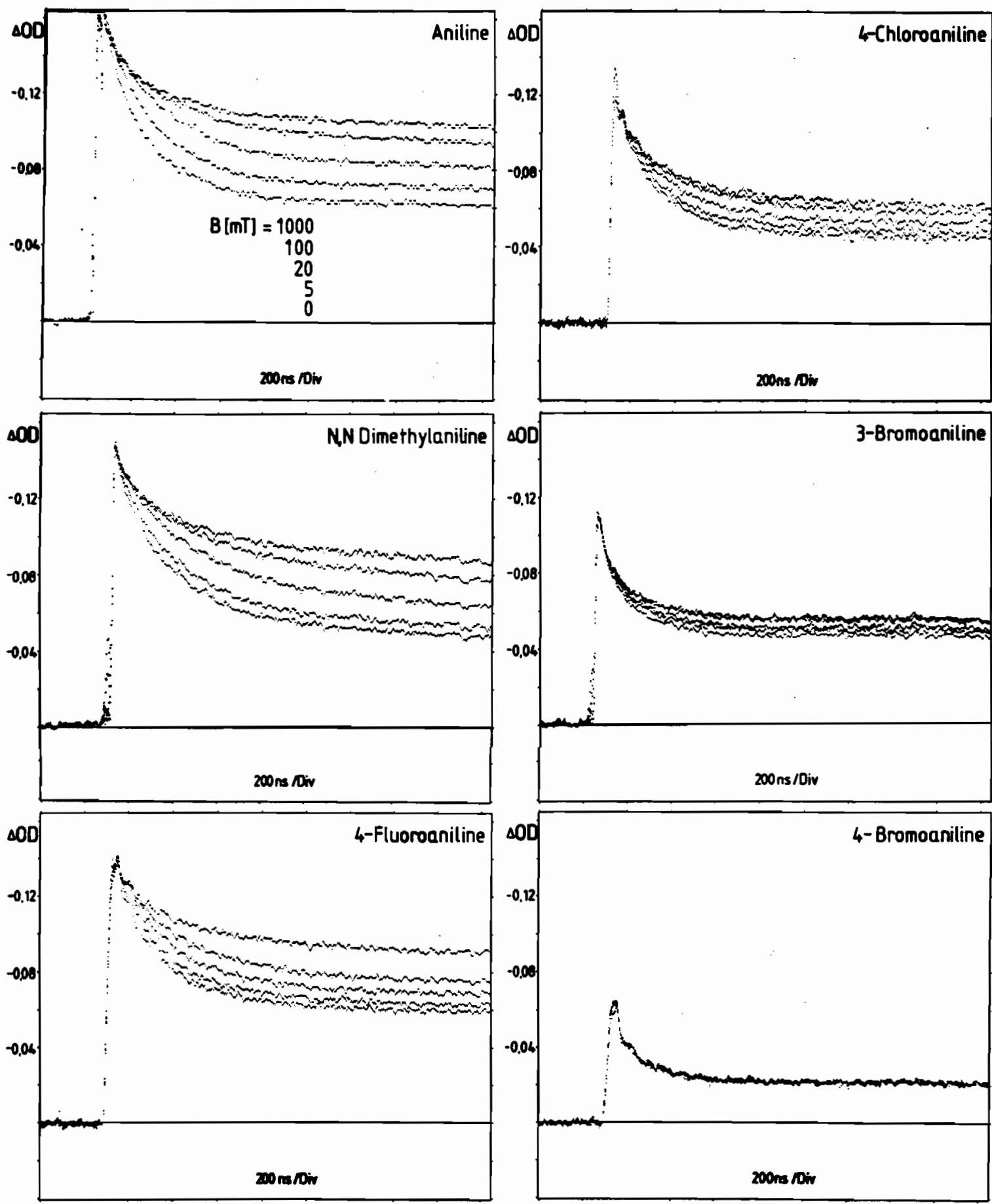

Fig. 5. Time dependence of absorption recovery observed at $600 \mathrm{~nm}$ under conditions of fast triplet quenching (cf. text) with various electron donors. The kinetic curves shown have been obtained with magnetic fields indicated in the aniline diagram. In each diagram the signal order from bottom to top corresponds to increasing magnetic field.

efficiency of triplets to radicals is high, since this does not go along with a marked absorption recovery, but if the geminate radical yield in the triplet quenching by heavy atom substituted donors gets lower, the triplet decay is accompanied by a larger absorbance change due to direct ground state repopulation in the triplet quenching and then the fast triplet decay kinetics becomes more apparent. The results given in Table 2 have been evaluated from the slower decay component. They would imply that the effective intramicellar radical pair recombination rate constant is very similar for all donors investigated.
The next point of interest is the influence of the magnetic field. There is a strong retardation of intramicellar radical pair recombination due to a magnetic field (cf. Table 2), which goes along with a concomitant increase of the yield of free radicals. For aniline, N,N-dimethylaniline and 4-fluoroaniline the magnetic field effect appears to be rather similar. In Fig. 6(a) we plotted the magnetic field dependence of the radical escape efficiency for these three donors. In fact, aniline and $\mathrm{N}, \mathrm{N}$-dimethylaniline show almost identical behaviour with an indication of saturation approach at $1 \mathrm{~T}$. Compared with these latter donors 

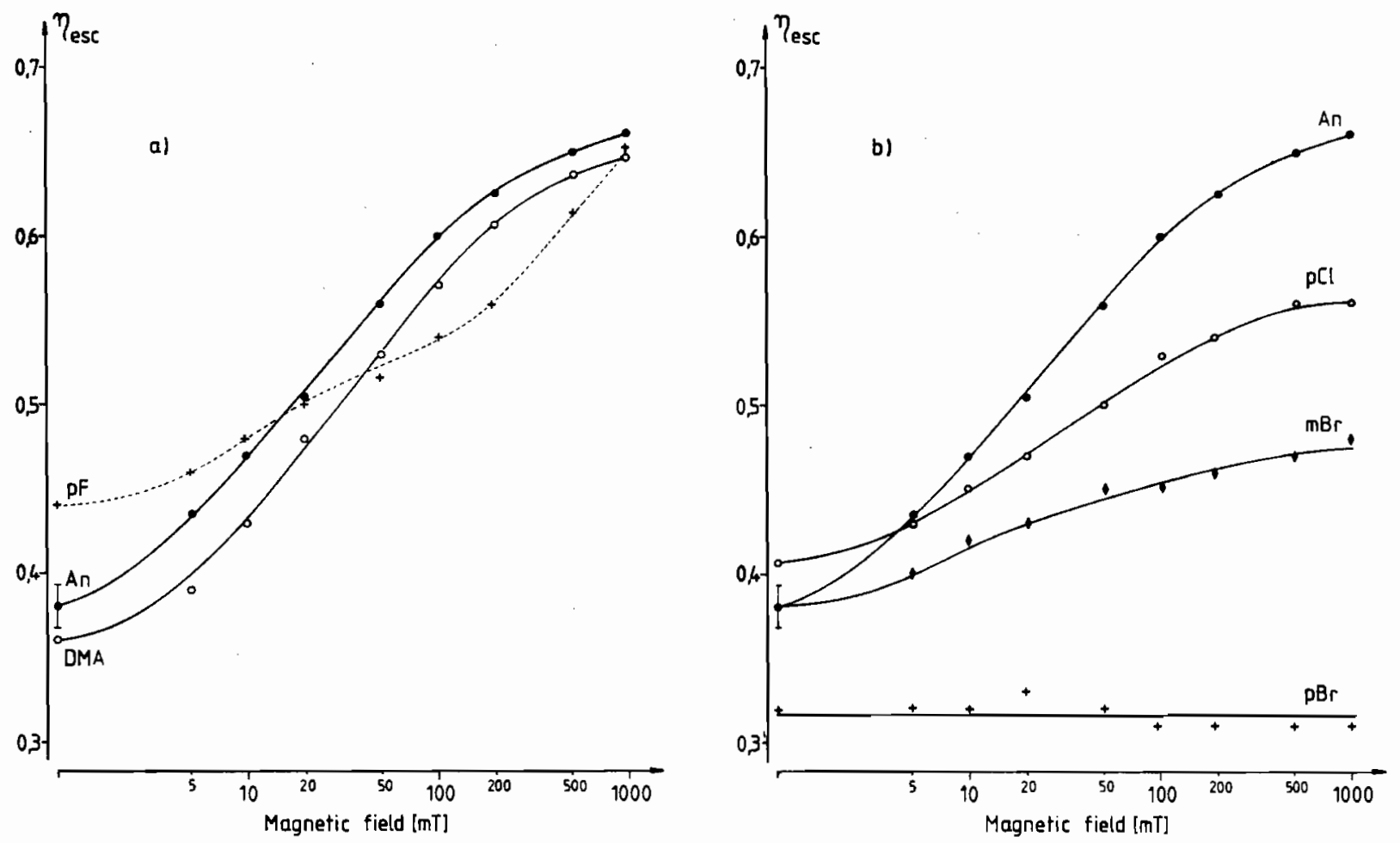

Fig. 6. Escape efficiency $\eta_{\mathrm{csc}}$ determined from the nanosecond absorption recovery signals at $600 \mathrm{~nm}$ (cf. Fig. 5) as the ratio of $\triangle O D$ at $2 \mu$ s to $\triangle O D$ in the maximum of the signal. (a) Comparison of the magnetic field dependence of $\eta_{\text {esc }}$ for aniline, N,N-dimethylaniline and 4-fluoroaniline. (b) Comparison of the series aniline, 4-chloro-, 3-bromo- and 4-bromoaniline.

4-fluoroaniline shows a less regular magnetic field dependence with a much slower (approximately logarithmic) saturation behaviour (note that the magnetic field is scaled logarithmically in Fig. 6). In the series aniline, 4-chloro-, 3-bromo- and 4-bromoaniline the extent of the magnetic field effect is more and more reduced (cf. Fig. 6(b)). It should be noted, that heavy atom attenuation of the magnetic field effect is practically the same when monitoring the escape efficiency, normalized to the amount of geminate radical pairs produced, as when monitoring the free radical yield, normalized to the amount of triplets from which the radicals are produced (Fig. 4). As is shown in Fig. 6(b) the halogen substituents do not noticeably influence the saturation behaviour and the half-field value.

\section{DISCUSSION}

Radical pair spin evolution and intramicellar recombination kinetics

Since the radical pairs are produced by electron transfer from a closed-shell donor to a triplet-excited acceptor they will originate with a triplet spin alignment. Immediate reverse electron transfer to form the energetically lower unreacted ground state donoracceptor pair is not possible because the final state is of singlet multiplicity. The detailed kinetic scheme to be considered for intramicellar radical pair recombination is depicted in Fig. 7. In zero magnetic field the radical pair may exist in four nearly degenerate spin states (one triplet and one singlet). By an external magnetic field the degeneracy of the triplet levels is lifted due to the Zeeman interaction. Only the singlet radical pair can undergo reverse electron transfer to yield the singlet ground state of the unreacted pair (UP). The spin substates of the radical pair can be converted into each other by several mechanisms to be discussed in more detail below.

Of course, the exact recombination kinetics of the radical pair derived from such a four-state scheme will in general not be a simple monoexponential decay. We will, however, not proceed with a quantitative analysis here (the kinetic scheme has been investigated in some detail by Hayashi and Nagakura ${ }^{29}$ ) but rather will qualitatively discuss some limiting cases providing most of the physical insight. Depending on the ratedetermining step, the effective rate constant of recombination $\left(k_{\mathrm{rec}}\right)$ will be approximated either by $k_{\mathrm{Ts}}$ or by $1 / 4 k_{\text {ret }}$

$$
k_{\mathrm{rec}} \approx \operatorname{Min}\left(k_{\mathrm{Ts}}, k_{\mathrm{ret}} / 4\right) \text {. }
$$

In zero field the triplet substates can be treated as kinetically equivalent. The effective rate constant of their transition to the singlet substate $\left(k_{\mathrm{TS}}\right)$ is made up of two contributions

$$
k_{\mathrm{TS}}=k_{\mathrm{IHF}}+k_{\mathrm{rel}} \text {. }
$$

(1) Isotropic hyperfine coupling is generally considered as the most important in the radical pair mechanism and is responsible for CIDNP. This interaction comes in as a time-independent contribution to the spin Hamiltonian and mixes triplet and singlet substates. Actually, this type of interaction produces coherent, reversible triplet-singlet transitions (cf. e.g. Ref. 26a) but, still, a characteristic time constant can 


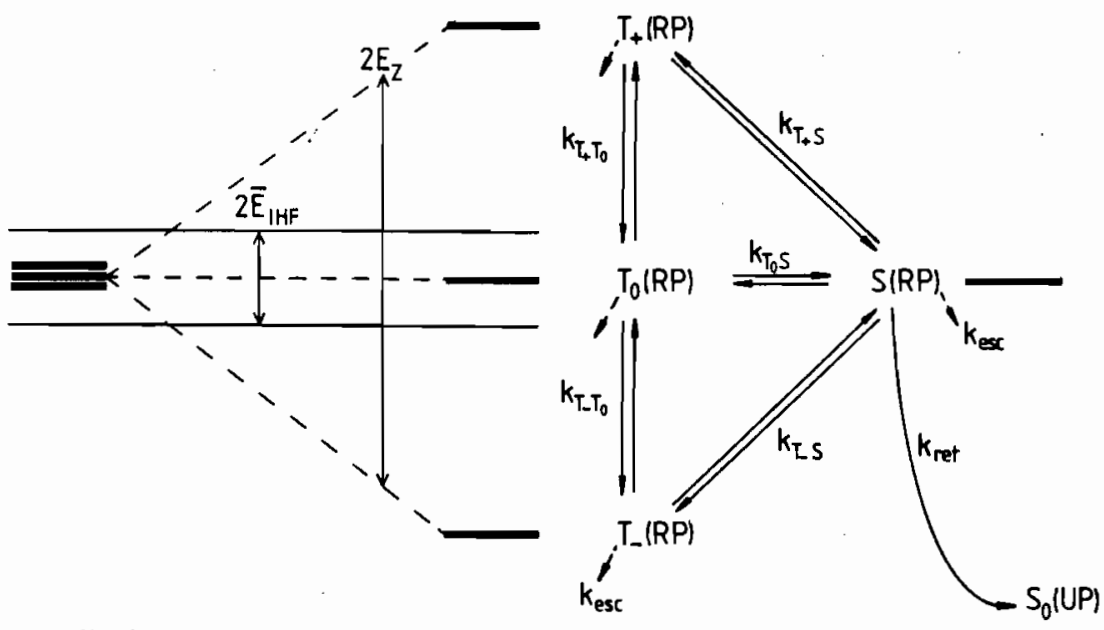

zero field

high field

Fig. 7. Scheme describing the kinetic relations between the spin states of the radical pair (RP) and the unreacted pair (UP), which is formed upon recombination. The short dashed arrows indicate the reaction channel of micellar escape, which is equally available for all spin substates. On the left-hand side is shown the energy level scheme for the triplet substates in zero external magnetic field and in high field. $\bar{E}_{\mathrm{iHF}}$ denotes an average hyperfine coupling energy corresponding to an internal magnetic field given by

$B_{1 / 2}(\mathrm{IHF})$ in Eq. (5). The Zeeman energy in the external magnetic field is given by $E_{\mathrm{z}}$.

be attributed to the resulting spin motion, the inverse of which may be used as an approximate first-order rate constant $k_{\mathrm{IHF}}$.

(2) Besides the isotropic hyperfine coupling, which is time-independent, there are several magnetic interactions, fluctuating in time (e.g. anisotropic hyperfine coupling and various spin-orbit coupling effects), which cause relaxation among the spin substates. ${ }^{33}$ Their contribution to the triplet-singlet intersystem crossing is collected in the rate constant $k_{\text {rel. }}$.

We have estimated the contribution $k_{\mathrm{IHF}}$ for the radical pairs investigated in this paper using the semiclassical method of Schulten and co-workers. ${ }^{26 c, d}$ The result is in the order of $2 \times 10^{8} \mathrm{~s}^{-1}$ which gives us the information that $k_{\mathrm{TS}} \geqslant 2 \times 10^{8} \mathrm{~s}^{-1}$. This value is much faster than the effective recombination rate constant $\left(3 \times 10^{6} \mathrm{~s}^{-1}\right)$, found experimentally in zero field. Therefore we conclude that the spin evolution of the triplet-born radical pair is not rate determining for the recombination process, i.e. we deal with that limiting kinetic case, where the effective first-order recombination rate constant is given by the equilibrium singlet probability $(1 / 4)$ times the specific rate constant $k_{\text {ret }}$ of reverse electron transfer in a singlet radical pair. As we have previously shown, ${ }^{28}$ this rate constant depends on the size of the waterpool of the micelle and is largely determined by the rate constant of diffusional encounters within the waterpool volume.

The interpretation given for the zero-field kinetics is corroborated by the magnetic field effects observed. Leaving aside for the moment the 4-iodoaniline case, it is found that the yield of free radicals increases with a magnetic field. It becomes clear from the diagrams in Fig. 5 that the magnetic field does not affect the primary yield of geminate radical pairs but develops during the time period of intramicellar recombination. The kinetic analysis according to Eq. (1) shows that $k_{\text {rec }}$ is slowed down by a magnetic field and, since $k_{\text {esc }}$ is essentially magnetic field-independent, the efficiency of escape, $\eta_{\text {esc }}$, given by Eq. (4), increases

$$
\eta_{\text {esc }}=k_{\text {esc }} /\left(k_{\text {esc }}+k_{\text {rec }}\right) \text {. }
$$

The effect of the magnetic field on the reaction kinetics may be understood in terms of Fig. 7. Due to the increasing Zeeman splitting of two of the triplet sublevels their kinetic behaviour will change. Here the partitioning (cf. Eq. (3)) of the rate constant $k_{\mathrm{Ts}}$ is of particular importance because $k_{\mathrm{IHF}}$ and $k_{\text {rel }}$ respond with quite different sensitivity to the increase of the Zeeman splitting. So far, most investigators of the radical pair mechanism have mainly concentrated on the magnetic field effect on the isotropic hyperfine contribution $\left(k_{\mathrm{IHF}}\right)$. It has been established by many theoretical and experimental investigations (cf. Ref. 3 ), that $k_{\mathrm{IHF}}$ is rapidly going to zero when the Zeeman splitting exceeds a radical pair-specific average value of the isotropic hyperfine coupling energy. A characteristic half-field value for this effect has been given by Weller et al. ${ }^{34}$

$$
B_{1 / 2}(\mathrm{IHF})=2\left(B_{1}^{2}+B_{2}^{2}\right) /\left(B_{1}+B_{2}\right)
$$

where the contributions $B_{1}$ and $B_{2}$ of the individual radicals are obtained by summing over the coupling nuclei in each radical separately

$$
B_{1(2)}=\sum_{i} I_{i}\left(I_{i}+1\right) a_{i}^{2}
$$

The $B_{1 / 2}$-values obtained from Eq. (5a) for the radical pairs of thionine with various anilines are listed in Table 2.

Comparing these theoretical values with the experimental $\boldsymbol{B}_{1 / 2}$-values it becomes clear, that quenching of the isotropic-hyperfine-coupling part of $k_{\mathrm{TS}}$ can contribute only little to the magnetic field effect observed in this work.

Referring again to Fig. 7 we can derive that the overall rate constant of recombination will be only sensitive to changes in $k_{\mathrm{TS}}$ if the $\mathrm{T} \rightarrow \mathrm{S}$ process becomes rate determining for recombination, more 
specifically, if it approaches a value given by

$$
k_{\mathrm{Ts}}\left(B_{1 / 2}\right) \simeq k_{\text {ret }}
$$

From the large $B_{1 / 2}$-values observed, it has to be concluded that even after the contribution of $k_{\mathrm{IHF}}$ to $k_{\mathrm{T}_{+} \mathrm{s}}$ or $k_{\mathrm{T}_{ \pm} \mathrm{T}_{0}}$ has been magnetically quenched (note that $k_{\mathrm{T}_{0} \mathrm{~s}}$ is either independent of a magnetic field or will even increase in case the radicals differ in their $g$ values), $k_{\mathrm{T}_{+} \mathrm{S}}$ and $k_{\mathrm{T}_{ \pm} \mathrm{T}_{0}}$ are larger than the rate constant $k_{\text {ret }}$ of spin-allowed reverse electron transfer in singlet radical pairs, i.e.

$$
k_{\text {rel }}\left(B_{1 / 2}(\mathrm{IHF})\right)>k_{\text {ret }} .
$$

Spin relaxation processes $\left(k_{\text {rel }}\right)$ are less sensitive to a magnetic field (cf. Refs 28 and 29) than the coherent spin evolution caused by the isotropic hyperfine coupling and therefore higher fields are necessary to achieve the condition

$$
k_{\text {rei }}\left(B_{1 / 2}\right) \simeq k_{\text {ret }} .
$$

Hayashi and Nagakura ${ }^{29}$ were the first to point out the role of spin relaxation for a quantitative understanding of the magnetic field effects observed on intramicellar recombination kinetics. + We hope that our contribution may help to elucidate the relation between the "normal" radical pair mechanism (where spin motion depends only on isotropic hyperfine coupling) and the "supercage" radical pair mechanism characteristic of the long cage times in micelles.

In order to explain the heavy atom substituent effect on the magnetic field dependent radical pair recombination, the rate constant $k_{\text {rel }}$ should be resolved into different contributions

$$
k_{\text {rel }}(B)=k_{\text {rel,AHC }}(B)+k_{\text {rel,So }}(B)+k_{\text {rel,So }}^{\prime}
$$

Electron spin relaxation is brought about by anisotropic interactions, randomly modulated by the rotational tumbling of the molecule and fluctuations in its environment. ${ }^{33}$ These include anisotropic hyperfine coupling $\left(k_{\mathrm{rel}, \mathrm{AHC}}(B)\right)$, anisotropy of the $g$-factor $\left(k_{\mathrm{rel}, \mathrm{so}}(B)\right)$ and spin-rotational coupling $\left(k_{\mathrm{rel}, \mathrm{so}}^{\prime}\right)$, the latter two being related to spin-orbit coupling effects. ${ }^{36}$ Whereas, however, the former two relaxation mechanisms are sensitive to a magnetic field the last one is not. Thus, by increasing the spin-orbit coupling by means of the heavy atom effect, $k_{\text {rel,so }}^{\prime}$ is increased which cannot be quenched by a magnetic field and therefore represents a lower bound of $k_{\text {rel }}$ even at high fields. Since the rate constant $k_{\text {rec }}$ cannot be reduced to a value smaller than $k_{\mathrm{rel}}(B)$ (if this is smaller than $k_{\text {rec }}(B=0)$ ) this magnetic field independent heavy atom contribution to $k_{\text {rel }}$ can acount for the decreasing magnetic field effect on $k_{\text {rec }}$ when heavy atoms are introduced.

The contribution of spin-rotational coupling to $k_{\text {rel,so }}^{\prime}$ of the radical pair can be estimated on the assumption that it is determined by the inverse of the longitudinal relaxation time $T_{1}$ of that radical moiety which is subject to the increased spin-orbit coupling by heavy atom substituents. In fact, each $\alpha-\beta$ spin flip of such a radical will bring the radical pair from $T_{+}$ or $T_{-}$to $T_{0}$ or $S\left(T_{0}\right.$ and $S$ will not have to be

†Estimates of the contribution of spin relaxation to the decay of spin correlation in geminate radical pairs have been first given by $\mathrm{B}$. Brocklehurst. ${ }^{35}$ distinguished because $T_{0}$ is in rapid spin equilibrium with $\mathrm{S}$ even at high magnetic fields). Atkins and Kivelson ${ }^{36}$ have derived the following expression for spinrotational relaxation

$$
T_{\perp}^{-1}=T_{2}^{-1}=\left(12 \pi r^{3}\right)^{-1}\left(\Delta g_{\|}^{2}+2 \Delta g_{\perp}^{2}\right) k T / \eta .
$$

Here $r$ is the effective hydrodynamic radius of the radical and $\eta$ the viscosity of its environment. The anisotropic components of the $g$-tensor are not exactly known for the halogenaniline cation radicals. However, a reasonable estimation of these can be made on the basis of the corresponding data for some 5-halouracil radicals ${ }^{37}$ taking into account the different spin densities in our cases. Assuming a value of $3 \AA$ for the hydrodynamic radius of the radicals and a viscosity of $1 \mathrm{cP}$, as would correspond to pure bulk water, the following values are obtained for $T_{1}^{-1}$ : 4-chloroaniline, $2 \times 10^{5} \mathrm{~s}^{-1} ; 3$-bromoaniline, $7 \times 10^{5} \mathrm{~s}^{-1}$; 4-bromoaniline, $3 \times 10^{6} \mathrm{~s}^{-1}$; and 4-iodoaniline, $1 \times 10^{7} \mathrm{~s}^{-1}$. These rate constants mark the lower limits to which $k_{\mathrm{T}_{ \pm} \mathrm{s}}$ and hence $k_{\mathrm{rec}}\left(\mathrm{T}_{ \pm}\right)$may be decreased by a magnetic field. The occurrence of sizeable magnetic field effects on $k_{\text {rec }}$ requires that $k_{\text {rel }}$ drops much lower than $k_{\text {rec }}(B=0)$ (cf. Eq. 8). Bearing in mind that $k_{\text {rec }}(B=0)$ is about $3 \times 10^{6} \mathrm{~s}^{-1}$ it is seen that for 4-chloro- and 3-bromoaniline $k_{\text {rel }}$ can still be made much smaller, though it is approaching this limit. However, for 4-bromoaniline it is equal and for 4-iodoaniline it is higher, so that the magnetic field effect on $k_{\text {rec }}$ is completely quenched. It should be emphasized that the heavy atom has no significant influence on $k_{\text {rec }}$ at zero field which corroborates the interpretation that in zero field $k_{\mathrm{Ts}}$ is not rate determining for recombination.

Results pertaining to the heavy atom effect on intramicellar radical pair recombination have also been reported by Turro et al. ${ }^{38}$ who investigated magnetic field effects on the cage reaction of photolyzed $p$ monosubstituted dibenzylketones. Comparing, for example, their data for the $\mathrm{CH}_{3}-, \mathrm{Cl}$ - and $\mathrm{Br}$-substituted derivatives with our present results, similar features are borne out: no heavy atom influence on the cage effect in zero field but a decrease of the magnetic field sensitivity of the cage effect with increasing spin-orbit coupling of the substituent. With the $\mathrm{Cl}$ substituent an additional magnetic field effect at very high field strength comes into play in Turro's case, which is attributed to the $\Delta g$-mechanism, providing magnetic field enhanced $\mathrm{T}_{0} \rightarrow \mathrm{S}$ intersystem crossing in the radical pair. We do not expect similar $\Delta g$-effects in our systems since even at zero field $k_{\mathrm{T}_{0} \mathrm{~S}}$ is much faster than $k_{\text {ret }}$ and hence increasing of $k_{\mathrm{T}_{0} \mathrm{~S}}$ by the $\Delta g$ effect should not affect the rate constant of recombination.

\section{Factorization of the free radical yield}

The free radical yield $\Phi_{\mathrm{fr}}$ obtained when quenching excited triplets in reversed micelles by electron transfer from electron donors, may be written as the product of the primary yield $\Phi_{\text {rp }}$ of geminate radical pairs and the efficiency $\eta_{\text {esc }}$, at which escape of one of the radicals from the micelle can compete with intramicellar recombination

$$
\Phi_{\mathrm{fr}}=\Phi_{\mathrm{rp}} \eta_{\text {esc }} \text {. }
$$

In the last section we discussed, how the second factor on the right-hand side of Eq. (11) is modified by 
a reversed micellar cage, magnetic fields and heavy atom substituents. Here we note that the first factor, $\Phi_{\mathrm{rp}}$, is also sensitive to the nuclear charge of the donor substituent. This effect in the micellar solution has been shown to correspond to the effect which has been previously observed in homogeneous solution ${ }^{11}$ as a marked, position dependent heavy atom effect on the free radical yield. It has been explained semiquantitatively by the spin-orbit coupling contribution of the heavy atom substituents on the intersystem crossing rate constant of a triplet exciplex assumed as the primary product of electron transfer quenching. Thus, for a micellar solvent, too, such triplet exciplexes seem to be important intermediates. In homogeneous solutions there has been a magnetic field dependent decrease of the free radical yield according to the triplet mechanism which was especially marked for the case of the 4-iodoaniline quencher. This type of magnetic field effect is also observed in micellar solution. Its half-field value of about $300-400 \mathrm{mT}$ and the negative sign of the effect are characteristic of the triplet mechanism.

\section{CONCLUSION}

Whereas the yield of escape from triplet originating radical pairs is about 1 if the electron donor-acceptor systems considered in this work are investigated in homogeneous, low viscous solution, it is significantly reduced in inverted micellar solutions due to the "supercage effect" $\dagger$ exhibited by the detergent layer confining the microscopic waterpools wherein the radicals are free to diffuse. In zero magnetic field the escape efficiency determined by the competition of escape and recombination (cf. Eq. 4), is little dependent on heavy atom substituents of variations of hyperfine coupling. This has been explained by the fact, that in zero field the triplet-singlet equilibration of the triplet-borne radical pair is faster than the spinallowed intramicellar radical pair recombination rate and hence not rate determining for recombination.

When applying an external magnetic field, tripletsinglet transitions from $T_{ \pm}$become slower and more and more rate determining for recombination which is thus slowed down. However, the characteristic halffield values observed are significantly higher than the field strength expected to suppress coherent tripletsinglet transitions induced by isotropic hyperfine coupling. This means that in weak fields of the order of the isotropic hyperfine coupling, incoherent spinrelaxation processes are still fast enough to achieve near spin equilibrium during the time intervals between successive radical pair re-encounters in the micelle. The half-field values of $20-30 \mathrm{mT}$ observed should be considered necessary for quenching $T_{ \pm}-S$ or $T_{ \pm}-T_{0}$ relaxation processes. Introduction of heavy atom substituents enhances the contribution of a magnetic field independent relaxation mechanism (spinrotational relaxation). This explains why the magnetic field sensitivity of recombination and hence of the yield of escape is reduced by the introduction of heavy atoms.

†The term "supercage" for the micellar cage has been coined by Turro (cf. Ref. 17).

\section{EXPERIMENTAL}

Materials. Thionine (Merck) was purified according to Ref. 39. Aniline (Baker), 4-fluoroaniline (Merck), 3-bromoaniline (Merck), and N,N-dimethylaniline (Ferak) were distilled under vacuum and stored under $\mathrm{N}_{2}$ or argon. 4Bromoaniline (Merck) and 4-iodoaniline (Fluka) were recrystallized two times from petroleum ether (b.p. $35-80^{\circ}$ ). 4 Chloroaniline (Fluka, puriss. $>99 \%$ ) was used without further purification.

Benzyldimethylhexadecylammonium chloride (cetyldimethylbenzylammonium chloride (CDBA)) was obtained from Fluka and was purified several times by fractionated recrystallization from acetic acid ethyl ester. A treatment with active carbon was applied before the first recrystallization.

Solvents used were benzene (Fluka puriss. p.a.) and water, deionized and doubly distilled in a quartz apparatus. In the micellar solutions the molar ratio of water to CDBA was 15 . The total dye concentration was $5 \times 10^{-6} \mathrm{~mol}^{-1}$. Before laser-flash investigations the solutions were flushed with $\mathrm{N}_{2}$ $\left(\mathrm{O}_{2}\right.$ content less than $\left.5 \mathrm{ppm}\right)$ for $45 \mathrm{~min}$ in order to remove $\mathrm{O}_{2}$.

To obtain reproducible results it was essential to obey the following procedure for preparing the micellar soln.

First CDBA was rapidly dissolved in benzene at $40^{\circ}$, then the proper amount of water and the aqueous stock solution of thionine was added. Although solubilization of the aqueous components seemed to be complete in a few minutes the soln was stirred for several hours at room temp. The donors were added in the appropriate dose of neat samples only shortly before the measurements. Precipitation of the detergent, eventually caused by too high local concentrations of the donors was avoided by stirring of the soln when adding the donors.

Laser-flash equipment. Experiments with microsecond time resolution were performed using a flash lamp pumped dye laser and a microprocessor-controlled kinetic flash spectrometer, which has been described elsewhere. ${ }^{13}$

The nanosecond time resolved experiments were carried out on a laser-flash spectrometer, schematically shown in Fig. 8. For laser excitation an excimer laser-pumped dye laser (using Rhodamin $6 \mathrm{G}$ as laser dye) of about $15 \mathrm{~ns}$ pulse width was used. The energy of the dye laser pulses was $2-3$ $\mathrm{mJ}$. The probe light beam (from a pulsed xenon arc lamp) and the exciting laser beam crossed in the cuvette at a small angle. A flow system, controlled by a magnetic valve, was used to replace reacted soln between any two exciting pulses. Normally 64 signals were stored and averaged on a transient digitizer (Tektronix 7912 AD). The synchronization and timing of the probe light pulse, trigger of transient digitizer and laser, shutter action and solution flow was achieved by a home-made electronic control circuit. The laser-flash spectrometer is described in detail in Ref. 40.

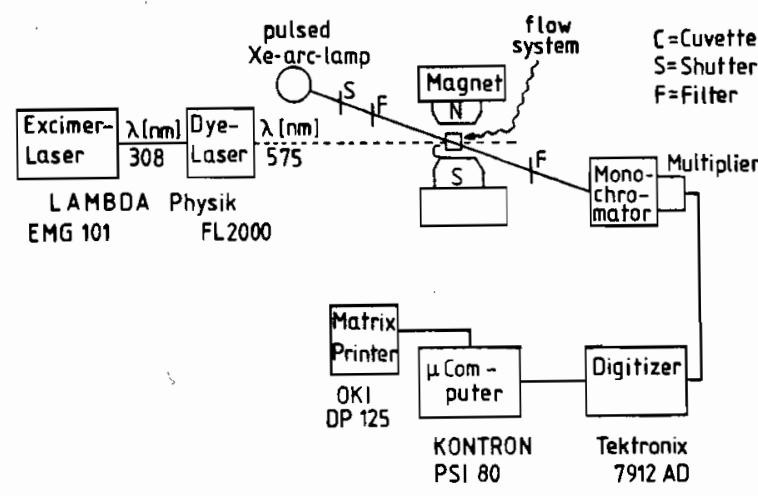

Fig. 8. Schematics of the nanosecond-laser-flash spectrometer. 
Acknowledgement-This work has been generously supported by the Deutsche Forschungsgemeinschaft. Financial support by the Fonds der Chemischen Industrie is also gratefully acknowledged.

\section{REFERENCES}

${ }^{1}$ A. Lepley and G. L. Closs (Editors), Chemically Induced Magnetic Polarization. Wiley, New York (1973).

${ }^{2}$ L. T. Muus, P. W. Atkins, K. A. McLauchlan and J. B. Pedersen (Editors), Chemically induced magnetic polarization, Proceedings of the NATO Advanced Study Institute, Sogesto Urbino, Italy. Reidel, Dordrecht (1977).

${ }^{3}$ K. M. Salikhov, Yu. N. Molin, R. Z. Sagdeev and A. L. Buchachenko, Spin polarization and magnetic field effects in radical reactions, Studies in Physical and Theoretical Chemistry (Edited by Yu. N. Molin), Vol. 22. Elsevier, Amsterdam (1984).

${ }^{4}$ P. W. Atkins and T. P. Lambert, Ann. Rep. Prog. Chem. A 72, 67 (1975).

${ }^{5}$ A. L. Buchachenko, Russ. Chem. Rev. (Usp. Khim.) 45 , 761 (1976).

${ }^{6}$ N. J. Turro, Proc. Nat. Acad. Sci. 80, 609 (1983); N. J. Turro and B. Kraeutler, Accts Chem. Res. 13, 369 (1980).

${ }^{7}$ K. H. Grellmann, A. R. Watkins and A. Weller, J. Luminescence 1, 2, 678 (1970).

${ }^{8}$ U. E. Steiner, G. Winter and H. E. A. Kramer, J. Phys. Chem. 81, 1104 (1977).

${ }^{9}$ T. Ohno, S. Kato, A. Yamada and T. Tanno, J. Phys. Chem. 87, 775 (1983); T. Ohno and N. N. Lichtin, J. Am. Chem. Soc. 102, 4636 (1980); T. Ohno and N. N. Lichtin, J. Phys. Chem. 86, 354 (1982); T. Ohno, S. Kato and N. N. Lichtin, Bull. Chem. Soc. Japan 55, 2753 (1982).

${ }^{10}$ P. Iwa, U. E. Steiner, E. Vogelmann and H. E. A. Kramer, J. Phys. Chem. 86, 1277 (1982).

${ }^{11}$ U. E. Steiner and G. Winter, Chem. Phys. Lett. 55, 364 (1978).

${ }^{12}$ G. Winter and U. E. Steiner, Ber. Bunsenges. Phys. Chem. 84, 1203 (1980).

${ }^{13}$ T. Ulrich, U. E. Steiner and R. E. Föll, J. Phys. Chem. 87, 1873 (1983).

${ }^{14}$ A. Weller, Z. Phys. Chem. NF 130, 129 (1982).

${ }^{15}$ W. Schlenker, T. Ulrich and U. E. Steiner, Chem. Phys. Lett. 103, 118 (1983).

${ }^{16}$ N. J. Turro and W. R. Cherry, J. Am. Chem. Soc. 100, 7431 (1978).

${ }^{17}$ Cf. N. J. Turro and G. C. Weed, J. Am. Chem. Soc. 105, 1861 (1983), and refs cited therein.

${ }^{18}$ Y. Sakaguchi, S. Nagakura and H. Hayashi, Chem. Phys. Lett. 72, 420 (1980); Y. Sakaguchi, H. Hayashi and S. Nagakura, J. Phys. Chem. 86, 3177 (1982); Y. Sakaguchi and H. Hayashi, Chem. Phys. Lett. 87, 539 (1982).

${ }^{19}$ N. J. Turto, M.-F. Chow, C.-J. Chung, Y. Tanimoto and G. C. Weed, J. Am. Chem. Soc. 103, 4574 (1981).

${ }^{20}$ Y. Tanimoto and M. Itoh, Chem. Phys. Lett. 83, 626 (1981); Y. Tanimoto, H. Udagawa and M. Itoh, J. Phys. Chem. 87, 724 (1983).

${ }^{21}$ J. C. Scaiano and E. B. Abuin, Chem. Phys. Lett. 81, 209
(1981) ; J. C. Scaiano, E. B. Abuin and L. C. Stewart, J. Am. Chem. Soc. 104, 5673 (1982).

${ }^{22}$ R. Kaptein and L. J. Oosterhoff, Chem. Phys. Lett. 4, 195 (1969); Ibid. 4, 214 (1969).

${ }^{23}$ G. L. Closs, J. Am. Chem. Soc. 91, 4552 (1969); G. L. Closs and A. D. Trifunac, Ibid. 92, 2183 (1970).

${ }^{24} \mathrm{~K}$. Schulten, H. Staerk, A. Weller, H.-J. Werner and B. Nickel, Z. Phys. Chem. NF 101, 371 (1976); H.-J. Werner, H. Staerk and A. Weller, J. Chem. Phys. 68, 2419 (1978); H. Schomburg, H. Staerk, A. Weller and H.-J. Werner Chem. Phys. Lett. 56, 399 (1978); F. Nolting, H. Staerk and A. Weller, Ibid. 88, 523 (1982); A. Weller, H. Staerk and R. Treichel, Faraday Discuss. Chem. Soc. 78, 271 (1984).

${ }^{25}$ M. E. Michel-Beyerle, R. Haberkorn, W. Bube, E. Steffens, H. Schröder, H. J. Neusser and E. W. Schlag, Chem. Phys. 17, 138 (1976); W. Bube, M. E. MichelBeyerle, R. Haberkorn and E. Steffens, Chem. Phys. Lett. 50, 389 (1977); M. E. Michel-Beyerle, H. W. Krüger, R. Haberkorn and H. Seidlitz, Chem. Phys. 42, 441 (1979); M. E. Michel-Beyerle, H. Scheer, H. Seidlitz, D. Tempus and R. Haberkorn, FEBS Lett. 100, 9 (1979); H. W. Krüger, M. E. Michel-Beyerle and H. Seidlitz, Chem. Phys. Lett. 87, 79 (1982)

${ }^{26 a} \mathrm{Z}$. Schulten and K. Schulten, J. Chem. Phys. 66, 4616 (1977); ${ }^{b}$ H.-J. Werner, Z. Schulten and K. Schulten, Ibid. 67, 646 (1977); ${ }^{c}$ K. Schulten and P. G. Wolynes, Ibid. 68 , 3292 (1978); ${ }^{d}$ E.-W. Knapp and K. Schulten, Ibid. 74, 1878 (1979); ${ }^{e}$ K. Schulten and R. Epstein, Ibid. 71, 309 (1979).

${ }^{27}$ R. Haberkorn, Chem. Phys. 19, 165 (1977); Ibid. 24, 111 (1977); Ibid. 26, 35 (1977).

${ }^{28}$ T. Ulrich and U. E. Steiner, Chem. Phys. Lett. 112, 365 (1984).

${ }^{29}$ H. Hayashi and S. Nagakura, Bull. Chem. Soc. Japan 57, 322 (1984).

${ }^{30}$ U. Klein and M. Hauser, Z. Phys. Chem. NF 90, 215 (1974); U. K. A. Klein, D. J. Miller and M. Hauser, Spectrochim. Acta 32A, 379 (1976); D. J. Miller, Dissertation, Universität Stuttgart (1977).

${ }^{31}$ H. Fischer, Z. Phys. Chem. NF 43, 12 (1964)

${ }^{32}$ E. J. Land and G. Porter, Trans. Faraday Soc. 59, 2027 (1963).

${ }^{33} \mathrm{Cf}$. treatises on ESR-spectroscopy, e.g. N. M. Atherton, Electron Spin Resonance. Wiley, New York (1973).

${ }^{34}$ A. Weller, F. Nolting and H. Staerk, Chem. Phys. Lett. 96, 24 (1983)

${ }^{35}$ B. Brocklehurst, Nature 221, 921 (1969); J. Chem. Soc. Faraday Trans. II 75, 123 (1979).

${ }^{36} \mathrm{P}$. W. Atkins and D. Kivelson, J. Chem. Phys. 44, 169 (1966).

${ }^{37}$ M. D. Sevilla, S. Swarts, H. Riederer and J. Hüttermann, J. Phys. Chem. 88, 1601 (1984).

${ }^{38}$ N. J. Turro, C.-J. Chung, G. Jones, II and W. G. Becker, J. Chem. Phys. 86, 3679 (1982).

${ }^{39}$ W. Endriss, Dissertation, Universität Stuttgart (1961).

${ }^{40} \mathrm{~T}$. Ulrich, Dissertation, Universität Konstanz (1986).

${ }^{41}$ H. P. Waschi, Dissertation, Universität Stuttgart (1983). 